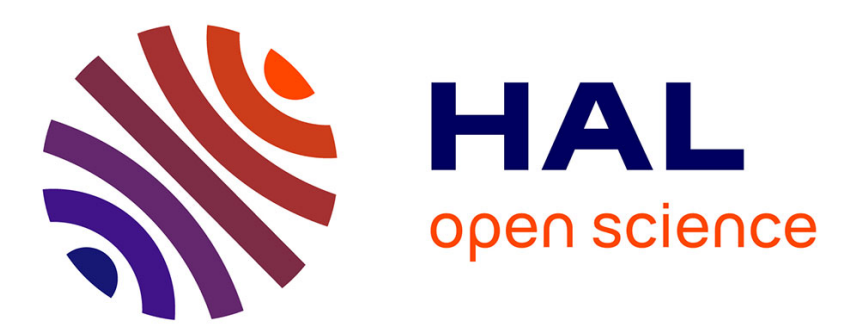

\title{
Température d'équilibre d'un collecteur. Plan dans des conditions réelles définies
}

\author{
J. Flechon
}

\section{To cite this version:}

J. Flechon. Température d'équilibre d'un collecteur. Plan dans des conditions réelles définies. Revue de Physique Appliquée, 1976, 11 (6), pp.743-750. 10.1051/rphysap:01976001106074300 . jpa00244110

\section{HAL Id: jpa-00244110 https://hal.science/jpa-00244110}

Submitted on 1 Jan 1976

HAL is a multi-disciplinary open access archive for the deposit and dissemination of scientific research documents, whether they are published or not. The documents may come from teaching and research institutions in France or abroad, or from public or private research centers.
L'archive ouverte pluridisciplinaire HAL, est destinée au dépôt et à la diffusion de documents scientifiques de niveau recherche, publiés ou non, émanant des établissements d'enseignement et de recherche français ou étrangers, des laboratoires publics ou privés. 
Classification

Physics Abstracts

7.500

\title{
TEMPÉRATURE D'ÉQUILIBRE D'UN COLLECTEUR PLAN DANS DES CONDITIONS RÉELLES DÉFINIES
}

\author{
J. FLECHON
}

Laboratoire de Physique de Dépôts Métalliques Université Nancy 1, 54037 Nancy Cedex, France

(Reçu le 24 mai 1976, accepté le 5 juillet 1976)

\begin{abstract}
Résumé. - On examine l'influence des facteurs climatiques : Global, température ambiante, vent et de la géométrie d'un insolateur : nombre de vitres, sur la température d'équilibre atteinte par le récepteur. On tient compte dans les calculs des rayonnements : Global et terrestre, de la convection interne, de la convection externe libre et forcée.
\end{abstract}

\begin{abstract}
The influence of climatic factors such as ambiant temperature, wind, insulator geometry and the number of windows on the equilibrium temperature reached by the collector are examined. Internal as well as external convection (free and forced) are taken into account in the calculation of the radiation.
\end{abstract}

Dans une précédente étude [1] limitée à l'intervention des phénomènes radiatifs nous avons, dans une ambiance tropicale $\theta_{\mathrm{amb}}=+27^{\circ} \mathrm{C}$, cherché à définir en fonction du nombre $\mathrm{n}$ de vitres la température d'équilibre limite $T_{\mathrm{e}}$ atteinte par un collecteur plan de capacité calorifique constante soumis à un rayonnement solaire direct, normal au plan de l'insolateur et supposé d'intensité constante.

Nous avons montré qu'au-delà d'un nombre de vitres $n=5, T_{\mathrm{e}}$ n'augmente plus et une investigation thermocinétique [2] prouve que la constante de temps $\tau$ devient supérieure aux durées compatibles avec un échauffement suffisant du récepteur si $n>3$. La loi suivie par sa température $T$ étant en première approximation

$$
\left(T_{\mathrm{e}}-T\right)=\left(T_{\mathrm{e}}-T_{\mathrm{amb}}\right) \mathrm{e}^{-t / \tau} .
$$

$\tau$ est proportionnel à $(n+1)$.

Nous tentons ici de généraliser ce premier travail en intégrant simultanément et en détaillant dans la mise en équation les paramètres initialement bloqués par l'introduction d'un terme correcteur $\varphi=0,9$ dans une ambiance donnée et empiriquement lié aux échanges thermiques parasites. Notre point de vue est différent de celui de J. A. Duffie et W. A. Beckman [3] qui écrivent l'équation de base d'un collecteur plan en termes généraux sans qu'un calcul de chaque terme soit précisé.

Cela nous permettra de définir pour le récepteur la température limite qu'en aucun cas il ne sera possible de dépasser.
Hypothèses. - L'insolateur protégé par $n=0,1,2$ 3 vitres reçoit un rayonnement solaire global $G=200,300, \ldots, 1000 \mathrm{~W} / \mathrm{m}^{2}$. Ses dimensions sont suffisantes ( $1 \mathrm{~m}^{2}$ au moins) pour que les effets de bord soient faibles. Les vitres sont supposées parfaitement transparentes pour le visible, parfaitement opaques pour l'infrarouge. Le collecteur est considéré comme un corps noir. Son isolation inférieure et latérale admise comme parfaite.

Les paramètres intervenant simultanément sur son équilibre thermique sont :

$1^{0}$ Le rayonnement solaire global $G$ dont on suppose qu'arrive au collecteur la quantité $G(1-r)^{2 n}, r$ : facteur de réflexion normale à chaque surface dioptrique.

$2^{\circ}$ Le rayonnement infrarouge émis par le collecteur supposé en équilibre thermique à la température $T_{\mathrm{e}}$ soit $\sigma T_{\mathrm{e}}^{4}$ (constante de Stéfan $\sigma=5,675 \times 10^{-8} \mathrm{~S}$. I.) et celui issu des vitres : la plus proche du collecteur ou première vitre $n=1$, étant à la température $T_{1}$, soit $\sigma T_{1}^{4}$ dans un demi-espace, la suivante $n=2 ; T_{2}, \sigma T_{2}^{4} \ldots$

$3^{\circ} \mathrm{La}$ convection interne ou chaleur émise par un plan à la température $T_{1}$ et reçue par un plan parallèle à la température $T_{2}$ distant du premier d'au moins 12 à $15 \mathrm{~m} / \mathrm{m}$ [4] $T_{1}>T_{2}$, l'énergie échangée étant en S. I. $\left(\mathrm{W} / \mathrm{m}^{-2}\right)$

$$
Q=1,23\left(T_{1}-T_{2}\right)^{1,25}=h\left(T_{1}-T_{2}\right)^{a}
$$

posant

$$
h=1,23 \quad \text { et } \quad a=\frac{5}{4} .
$$


$4^{\circ}$ Les échanges parasites intéressant la vitre externe et qui conditionnent l'équilibre thermique de l'ensemble. Ils sont de deux sortes :

a) Rayonnement terrestre : infrarouge entièrement absorbé par la vitre externe à la température $T_{\text {ve }}$. Son intensité est liée à l'ambiante $T_{\mathrm{a}}$ et à l'état du ciel. Supposons un ciel clair. Il rayonne comme un corps noir $(\varepsilon=1)$ à une température inférieure d'environ $20^{\circ} \mathrm{C}$ à celle de l'ambiante [4]. Ceci s'écrit $Q_{\mathrm{t}}=\sigma\left(T_{\mathrm{a}}-20\right)^{4}$, mais nous pouvons dans un but de simplification des calculs, considérer le ciel comme un corps gris d'émissivité $\varepsilon_{1}<$ supposé à $T_{\mathrm{a}}$ ce qui s'écrit :

$\underset{\left.(\mathrm{w} \mathrm{m})^{-2}\right)}{Q_{\mathrm{t}}}=\varepsilon_{1} \sigma T_{\mathrm{a}}^{4}=\sigma\left(T_{\mathrm{a}}-20\right)^{4}$ soit $\varepsilon_{1}=\left(1-\frac{20}{T_{\mathrm{a}}}\right)^{4}$

pour $T_{\mathrm{a}}$ variant de $270 \mathrm{~K}$ à $300 \mathrm{~K}$ nous prendrons $\varepsilon_{1}=0,76$.

b) Echanges convectifs externes : ils n'intéressent que le vitrage au contact avec l'air ambiant. Ce dernier peut être au repos : la convection est dite libre et la chaleur perdue

$$
Q_{\mathrm{c}_{1}}=b\left(T_{\mathrm{ve}}-T_{\mathrm{a}}\right)^{2}
$$

avec en S. I.

$\left(\mathrm{w} \mathrm{m}^{-2}\right)$

$$
b=2, \quad a=\frac{5}{4} .
$$

Si le vent souffle parallèlement au récepteur le régime d'écoulement est turbulent et la convection forcée.

Retenons 2 cas particuliers pour la vitesse du vent soit $v_{1}=3 \mathrm{~m} / \mathrm{s}$, la chaleur perdue dans ces conditions [3]

$$
Q_{\mathrm{cf}_{1}}=10 b\left(T_{\mathrm{ve}}-T_{\mathrm{a}}\right)
$$

et $v_{2}=10 \mathrm{~m} / \mathrm{s}$

$$
Q_{\mathrm{cf}_{2}}=32,5 b\left(T_{\mathrm{ve}}-T_{\mathrm{a}}\right) .
$$

1. Mise en équation. - Ayant recensé l'ensemble des paramètres réels intervenant dans l'équilibre thermique de l'insolateur, il suffit d'écrire pour chacun de ses éléments que la chaleur reçue est égale à la chaleur perdue en mettant à part le cas de la vitre externe.

a) Le collectéur :

$$
G(1-r)^{2 n}+\sigma T_{1}^{4}=\sigma T_{\mathrm{e}}^{4}+h\left(T_{\mathrm{e}}-T_{1}\right)^{a} .
$$

b) La $1^{\text {re }}$ vitre :

$\sigma\left(T_{\mathrm{e}}^{4}+T_{2}^{4}\right)+h\left(T_{\mathrm{e}}-T_{1}\right)^{a}=2 \sigma T_{1}^{4}+h\left(T_{1}-T_{2}\right)^{a}$.

De proche en proche, on passe à :

c) La vitre externe :

Elle reçoit le rayonnement terrestre, celui de la vitre voisine et la convection interne de celle-ci.

Elle émet sur ses 2 faces et perd de la chaleur par convection externe libre ou forcée

$$
\begin{array}{rr}
\sigma T_{(\mathrm{ve}-1)}^{4}+\varepsilon_{1} \sigma T_{\mathrm{a}}^{4}+h\left(T_{\mathrm{ve}-1}-T_{\mathrm{ve}}\right)^{a}=2 \sigma T_{\mathrm{ve}}^{4}+\quad \begin{array}{r}
b\left(T_{\mathrm{ve}}-T_{\mathrm{a}}\right)^{a} \\
\text { air calme }
\end{array} \\
\begin{array}{rr}
\sigma T_{(\mathrm{ve}-1)}^{4}+\varepsilon_{1} \sigma T_{\mathrm{a}}^{4}+h\left(T_{\mathrm{ve}-1}-T_{\mathrm{ve}}\right)^{a}=2 \sigma T_{\mathrm{ve}}^{4}+10 & b\left(T_{\mathrm{ve}}-T_{\mathrm{a}}\right) \\
\text { vent } 3 \mathrm{~m} / \mathrm{s} \\
\sigma T_{(\mathrm{ve}-1)}^{4}+\varepsilon_{1} \sigma T_{\mathrm{a}}^{4}+h\left(T_{\mathrm{ve}-1}-T_{\mathrm{ve}}\right)^{a}=2 \sigma T_{\mathrm{ve}}^{4}+32,5 & b\left(T_{\mathrm{ve}}-T_{\mathrm{a}}\right) . \\
\text { vent } 10 \mathrm{~m} / \mathrm{s}
\end{array}
\end{array}
$$

d) Transformation des équations :

Cas de 3 vitres

$$
\begin{aligned}
\frac{G(1-r)^{2 n}}{\sigma}+T_{1}^{4} & =T_{\mathrm{e}}^{4}+\frac{h}{\sigma}\left(T_{\mathrm{e}}-T_{1}\right)^{a} \\
\frac{h}{\sigma}\left(T_{\mathrm{e}}-T_{1}\right)^{a}+T_{\mathrm{e}}^{4}+T_{2}^{4} & =2 T_{1}^{4}+\frac{h}{\sigma}\left(T_{1}-T_{2}\right)^{a} \\
\frac{h}{\sigma}\left(T_{1}-T_{2}\right)^{a}+T_{1}^{4}+T_{3}^{4} & =2 T_{2}^{4}+\frac{h}{\sigma}\left(T_{2}-T_{3}\right)^{a} \\
\frac{h}{\sigma}\left(T_{2}-T_{3}\right)^{a}+T_{2}^{4}+\varepsilon_{1} T_{\mathrm{a}}^{4} & =2 T_{3}^{4}+\frac{b}{\sigma}\left(T_{3}-T_{\mathrm{a}}\right)^{a}
\end{aligned}
$$


$(\alpha)$ terme pouvant prendre les formes $(\beta)$ ou $(\gamma)$, ce qui devient

$$
\begin{aligned}
\frac{G(1-r)^{2 n}}{\sigma} & =T_{\mathrm{e}}^{4}-T_{1}^{4}+\frac{h}{\sigma}\left(T_{\mathrm{e}}-T_{1}\right)^{a} \\
(1)+(2) & =T_{1}^{4}-T_{2}^{4}+\frac{h}{\sigma}\left(T_{1}-T_{2}\right)^{a} \\
(1)+(2)+(3) & =T_{2}^{4}-T_{3}^{4}+\frac{h}{\sigma}\left(T_{2}-T_{3}\right)^{a} \\
(1)+(2)+(3)+(4) & =T_{3}^{4}-\varepsilon_{1} T_{\mathrm{a}}^{4}+\frac{b}{\sigma}\left(T_{3}-T_{\mathrm{a}}\right)^{a}
\end{aligned}
$$

( 3 cas possibles $8 b, 8 c)$.

2. Résolution numérique : Emploi du calcul automatique. - Nous avons à résoudre le système (5), (6), (7), $(8 a, b, c)$ dans le cas où :

$$
\begin{aligned}
& n=0 ; 1 ; 2 ; 3 \\
& T_{\mathrm{a}}=270 ; 280 ; 290 ; 300 \mathrm{~K} \\
& G=200 \ldots 1000 \mathrm{~W} / \mathrm{m}^{2} .
\end{aligned}
$$

Examinons dans le cas où $n=3$ comment se présente le système. L'inconnue est $T_{\mathrm{e}}$ qui se situe obligatoirement entre $250 \mathrm{~K}$ et $500 \mathrm{~K}$.

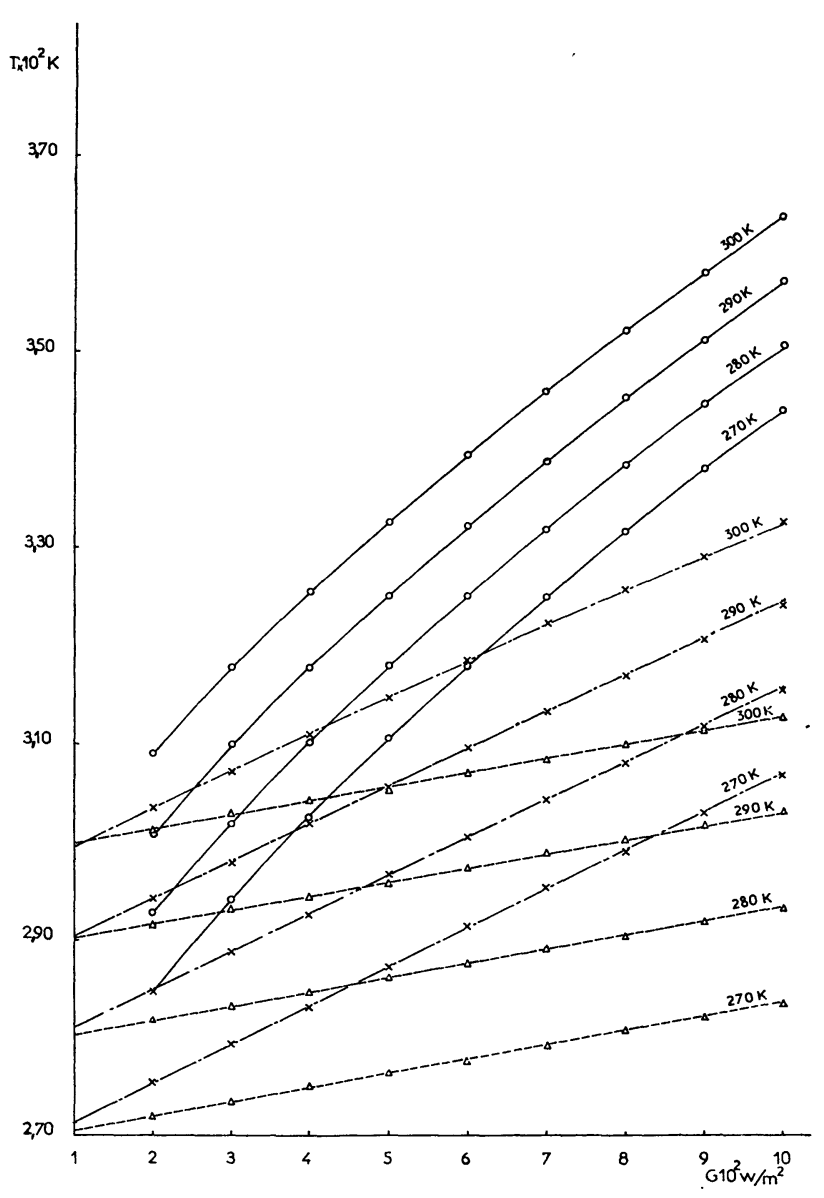

Fig. 1. - Température d'équilibre d'un insolateur. Plan (corps noir) horizontal ( 0 vitre) en fonction du rayonnement global $G$. - - - pas de vent. - $\times$. - Vent $3 \mathrm{~m} / \mathrm{s} . \cdots \Delta \cdots .$. Vent $10 \mathrm{~m} / \mathrm{s}$.
Tenant compte des puissances de $T$ et des valeurs de $\sigma$, nous allégeons le calcul en exprimant :

$T$ en centaines de $\mathrm{K}$, soit

$$
T_{\mathrm{a}}=2,7 ; 2,8 ; 2,9 ; 3
$$

$G$ en centaines de $\mathrm{W} / \mathrm{m}^{2}$, soit

$$
G=2 \ldots 10 \text {. }
$$

L'équation (8a) devient par exemple :

$$
13,75 G=T_{3}^{4}-0,76 \cdot T_{\mathrm{a}}^{4}+111,5\left(T_{3}-T_{\mathrm{a}}\right)^{5 / 4}
$$

équation du $16^{\mathrm{e}}$ degré où $T_{3}$ est l'inconnue.

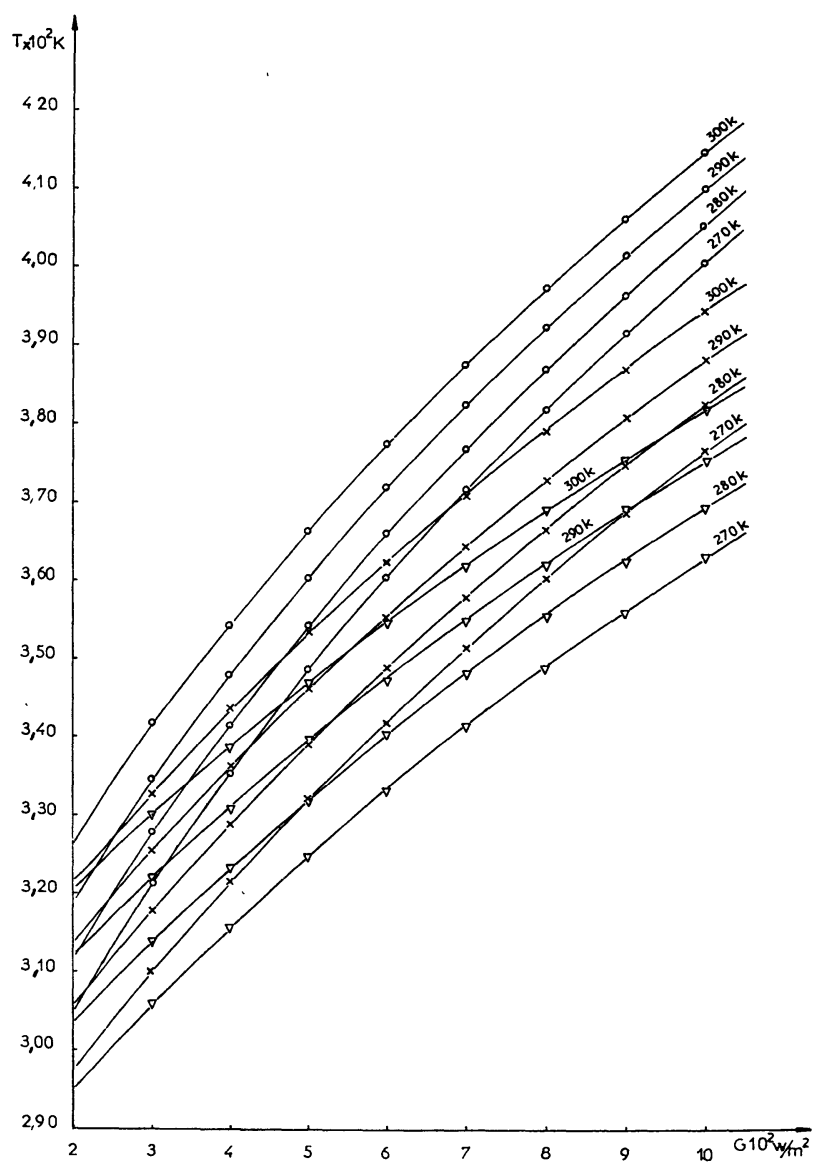

Fig. 2. - Température d'équilibre d'un insolateur. Plan horizontal (1 vitre) (corps noir) en fonction du rayonnement global G. -O- Pas de vent. $-\times-$ Vent $3 \mathrm{~m} / \mathrm{s}$. $-\Delta-$ Vent $10 / \mathrm{ms}$. 


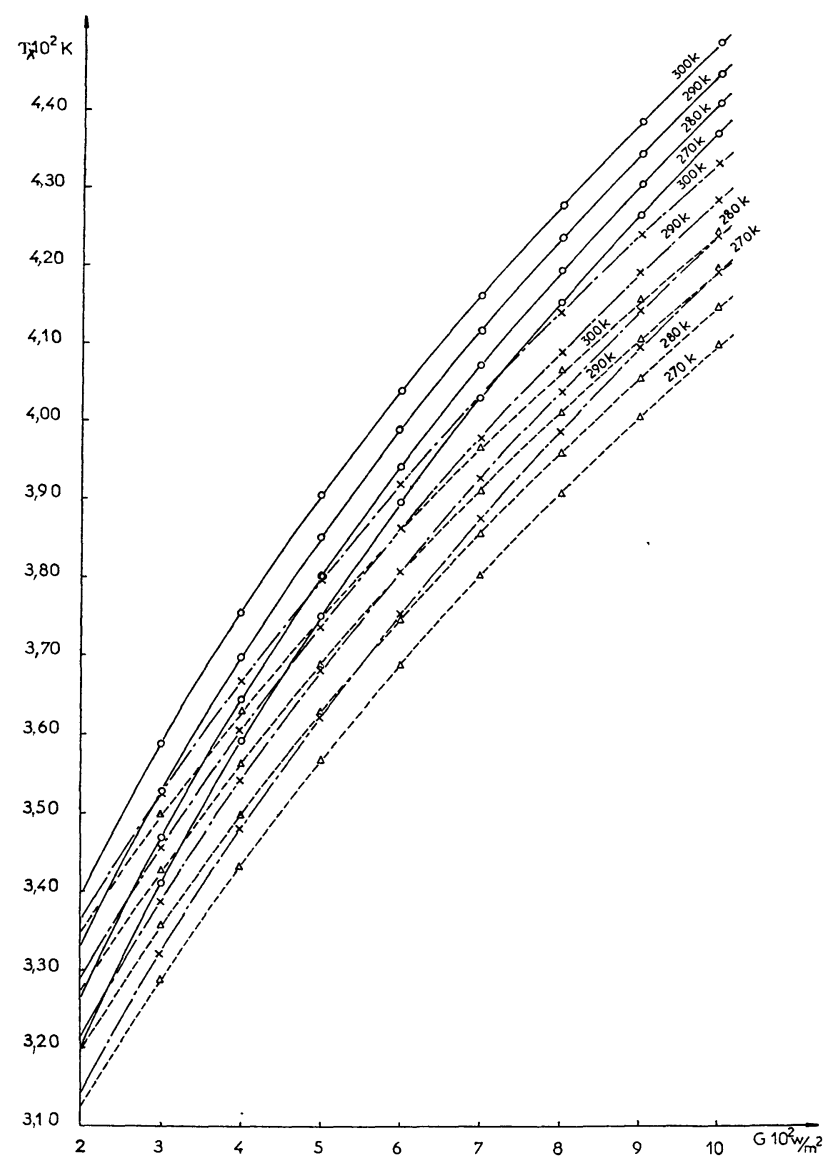

FIG. 3. - Température d'équilibre d'un insolateur. Plan horizontal (2 vitres) (corps noir) en fonction du rayonnement global G. -O- Pas de vent. - $\times$ - Vent $3 \mathrm{~ms}$. $\cdots \Delta \cdots$ Vent $10 \mathrm{~ms}$.

Deux méthodes de recherche des racines sont possibles : soit la méthode itérative de Newton, soit une recherche directe de l'ensemble des racines par la mźthode de Bairstow. Nous devons l'ensemble des calculs à M. Potevin de l'I. U. C. A. (U. E. R. de Mathématiques) de l'Université de Nancy I.

L'ordinateur donne sous forme de 4 tableaux de nombres en fonction de $G$ et de $T_{\mathrm{a}}$ les valeurs de $T_{3}, T_{2}$, $T_{1}, T_{\mathrm{e}}$ pour des vitesses du vent de $0,3 \mathrm{~m} / \mathrm{s}, 10 \mathrm{~m} / \mathrm{s}$. L'ensemble peut être résumé par des diagrammes explicitant sous forme de réseau $T_{\mathrm{e}}=f(G)$ (Fig. 1, 2, $3,4)$.

3. Résultats. - 3.1 OBSERVATIONS QUALITATIVES. L'effet de serre c'est-à-dire l'élévation de la température d'équilibre $T_{\mathrm{e}}$ de l'absorbeur au-dessus de l'ambiante est d'autant plus marqué qu'augmente le nombre de vitres.

En l'absence de vent la pente moyenne des courbes $T_{\mathrm{e}}=f(G)$ croît avec $n$, ainsi que leur courbure orientée vers les $G$ élevés et les basses températures. Si $n=0$, l'approximation de la droite est acceptable.

L'influence du vent sur $T_{\mathrm{e}}$ est d'autant plus faible que $n$ augmente. Celle des variations de l'ambiante $T_{\mathrm{a}}$ intervient dans le même sens : les courbes correspon-

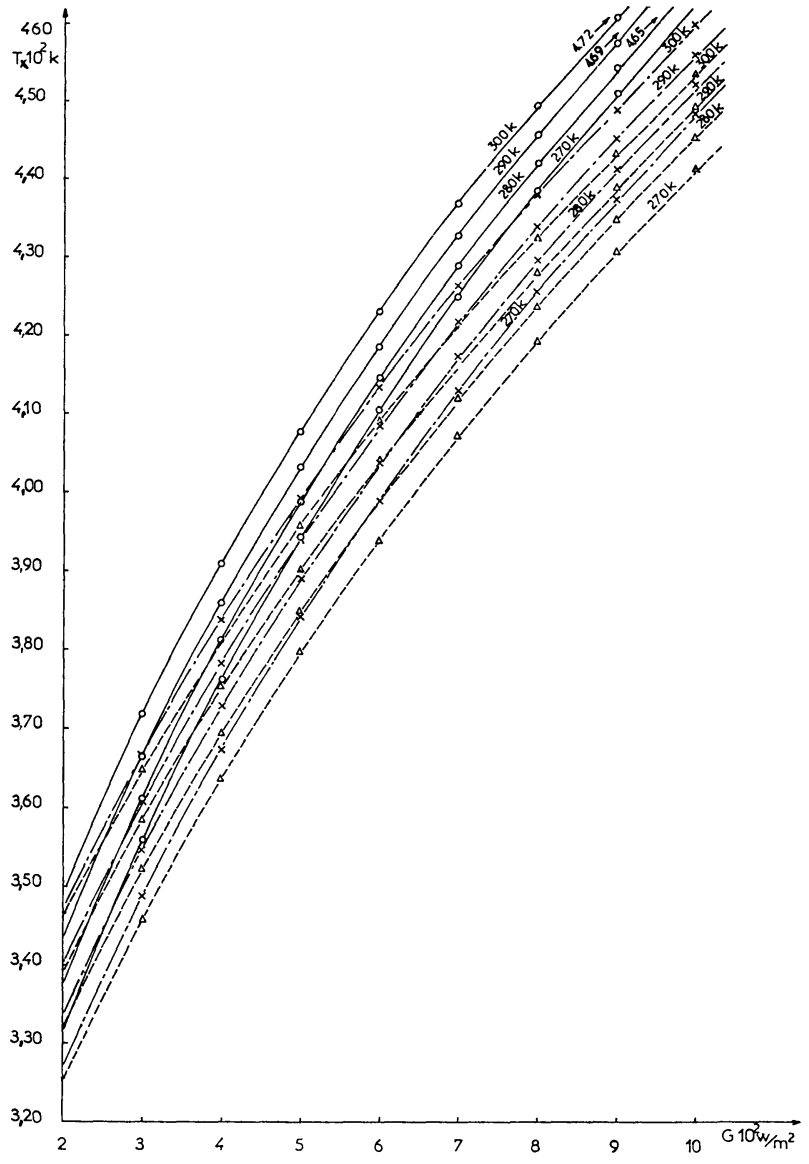

FIG. 4. - Température d'équilibre d'un insolateur. Plan horizontal (3 vitres) (corps noir) en fonction du rayonnement global G. $-\mathrm{O}-$ Pas de vent. - $\times$ - Vent $3 \mathrm{~m} / \mathrm{s} . \cdots \Delta \cdots$. Vent $10 \mathrm{~m} / \mathrm{s}$.

dant à des $\Delta T_{\mathrm{a}}=10^{\circ} \mathrm{C}$ se rapprochent si $n$ s'élève, ainsi qu'aux fortes valeurs de $G$.

3.2 OBSERVATIONS QUANTITATIVES. - Le tableau I résume pour 2 valeurs très différentes de $G$ (faible : $200 \mathrm{~W} / \mathrm{m}^{2}$ ) (fort : $900 \mathrm{~W} / \mathrm{m}^{2}$ ) les températures d'équilibre atteintes par le collecteur pour $n=0 ; 1 ; 2 ; 3$; 4 dans des ambiances variant de $-3^{\circ} \mathrm{C}$ à $+27^{\circ} \mathrm{C}$ sous des vents de $0 ; 3 ; 10 \mathrm{~m} / \mathrm{s}$.

Le tableau II souligne dans les mêmes conditions en prenant comme référence le corps noir directement exposé au rayonnement global le gain en température introduit par l'emploi de vitres.

Le tableau III montre comment le vent réduit les performances que l'on peut attendre en air calme.

4. Discussion. - PAS DE vITRE. - La température ambiante est d'autant plus déterminante sur le comportement $\mathrm{du}$ récepteur que le nombre de vitres diminue et que le rayonnement global est faible : la température d'un collecteur nu en équilibre thermique, chute de $15^{\circ}$ si l'ambiante diminue de $20^{\circ}\left(G=200 \mathrm{~W} / \mathrm{m}^{2}\right)$ sans vent, de $13^{\circ}$ seulement si $G=900 \mathrm{~W} / \mathrm{m}^{2}$ dans les mêmes conditions.

Elle chute également sous l'influence du vent d'autant plus que l'ambiante est faible et que le global croît. 
TABLEAU I

Températures d'équilibre $T_{\mathrm{e}}$ du collecteur Faibles G $\left(200 \mathrm{~W} / \mathrm{m}^{2}\right)$

\begin{tabular}{|c|c|c|c|c|c|c|c|c|c|}
\hline & & & & & & & & & \\
\hline & Vitres & 0 & 1 & 2 & 3 & 0 & 1 & 2 & 3 \\
\hline$\theta_{\mathrm{anib}}$ & & & & & & & & & \\
\hline $27^{\circ} \mathrm{C}$ & vent 0 & 309 & 327 & 340 & 350 & 358 & 406 & 438 & 461 \\
\hline & vent $3 \mathrm{~m} / \mathrm{s}$ & 303 & 322 & 337 & 348 & 329 & 387 & 424 & 449 \\
\hline & vent $10 \mathrm{~m} / \mathrm{s}$ & 301 & 321 & 335 & 346 & 311 & 375 & 416 & 443 \\
\hline $17^{\circ} \mathrm{C}$ & vent 0 & 301 & 319 & 333 & 344 & 351 & 401 & 434 & 458 \\
\hline & vent $3 \mathrm{~m} / \mathrm{s}$ & 294 & 314 & 329 & 341 & 320 & 381 & 419 & 445 \\
\hline & vent $10 \mathrm{~m} / \mathrm{s}$ & 291 & 312 & 328 & 340 & 301 & 369 & 410 & 439 \\
\hline $7^{\circ} \mathrm{C}$ & vent 0 & 293 & 312 & 327 & 338 & 345 & 396 & 430 & 454 \\
\hline & vent $3 \mathrm{~m} / \mathrm{s}$ & 285 & 306 & 322 & 334 & 312 & 375 & 414 & 441 \\
\hline & vent $10 \mathrm{~m} / \mathrm{s}$ & 282 & 304 & 320 & 333 & 292 & 362 & 405 & 434 \\
\hline
\end{tabular}

TABLEAU II

Gain sur $T_{\mathrm{e}}$ dû aux vitres

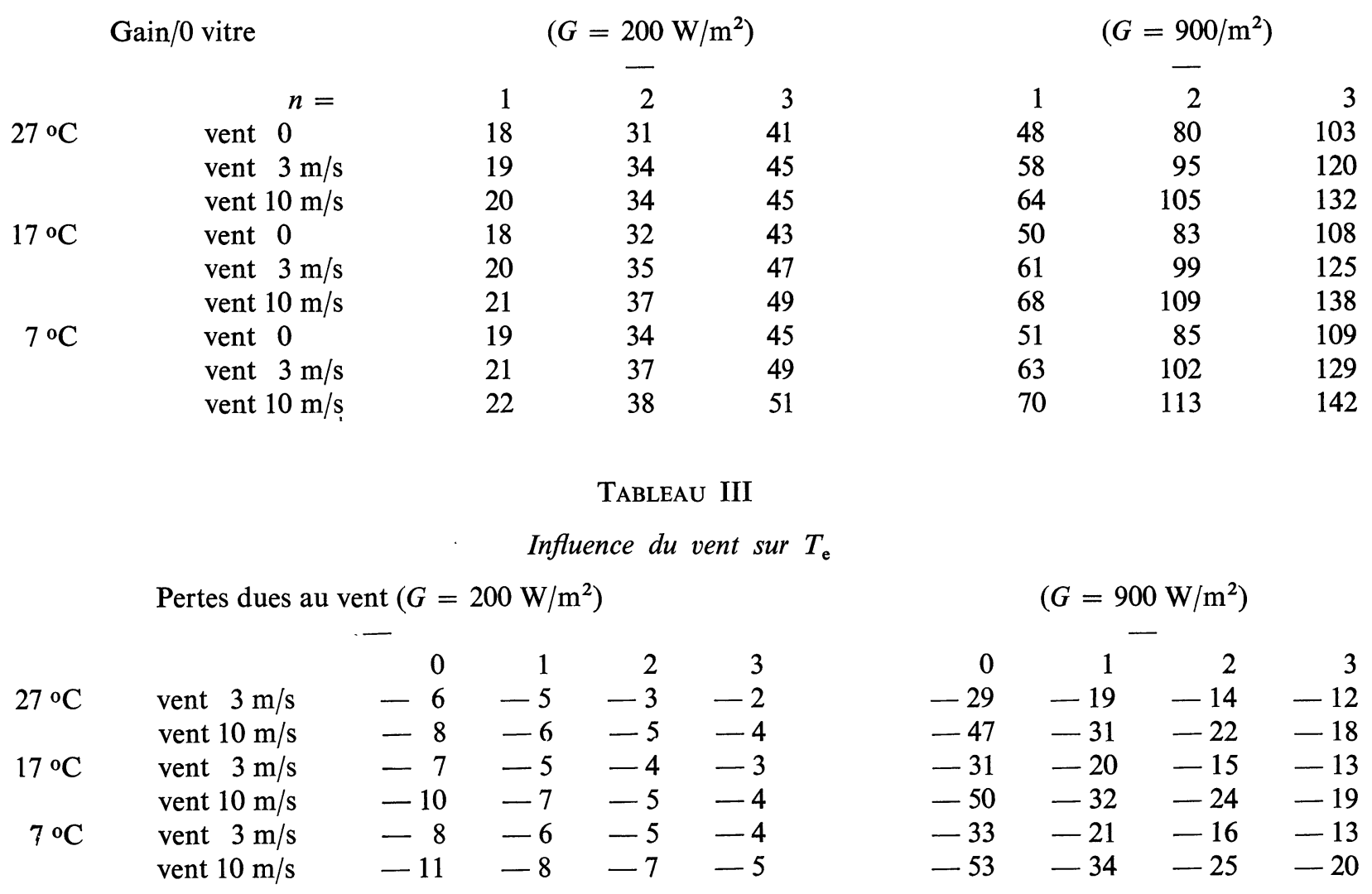

INFLUENCE DES VITRES. - L'effet de serre se manifeste aux basses puissances $\left(G=200 \mathrm{~W} / \mathrm{m}^{2}\right)$ par un gain d'environ $20^{\circ}$ lorsqu'on emploie une vitre, de $30^{\circ}$ à $35^{\circ} \mathrm{C}$ avec 2 vitres, de $40^{\circ}$ à $45^{\circ} \mathrm{C}$ si le collecteur comporte 3 vitres. Les gains deviennent respectivement 50 à $70^{\circ} \mathrm{C}, 80$ à $110^{\circ} \mathrm{C}$ et 100 à $140^{\circ} \mathrm{C}$ aux puissances élevées $\left(G=900 \mathrm{~W} / \mathrm{m}^{2}\right)$. Ce qui, avec deux vitres permet de se référer à une température d'équilibre de $157^{\circ} \mathrm{C}$ pour une ambiante de $7^{\circ} \mathrm{C}$, s'il n'y a pas de vent et de $132^{\circ} \mathrm{C}$ sous un vent de $10 \mathrm{~m} / \mathrm{s}$.
L'intérêt de la $2^{\mathrm{e}}$ vitre est évident car dans les mêmes conditions une vitre seulement conduit respectivement à $123^{\circ} \mathrm{C}$ et $89^{\circ} \mathrm{C}$ les températures limites du collecteur. Ces dernières sont assez peu dépendantes de l'ambiante étant donnée la protection assurée par le vitrage. Si $\theta_{\mathrm{e}}$ s'élève de $20^{\circ} \mathrm{C}$ soit $27^{\circ} \mathrm{C}$ la limite n'augmente pas plus de 8 à $10^{\circ} \mathrm{C}$ et inversement le refroidissement extérieur ne fait pas chuter d'autant la température $\mathrm{du}$ récepteur. $\mathrm{Ce}$ point est essentiel : en effet, si l'ambiante passe de $+27^{\circ} \mathrm{C}$ à $-3^{\circ} \mathrm{C}$, l'équilibre du 
collecteur protégé par 2 vitres passe pour $G=800 \mathrm{~W} /$ $\mathrm{m}^{2} \mathrm{de} 165^{\circ} \mathrm{C}$ à $153^{\circ} \mathrm{C}$ en l'absence de vent soit une diminution de $12{ }^{\circ} \mathrm{C}$ pour un refroidissement extérieur de $30^{\circ} \mathrm{C}$. Notons, qu'au moins en théorie, l'emploi de 3 vitres accentue considérablement ces avantages température limite plus haute et rendue moins sensible aux perturbations extérieures. Mais interviennent dans la réalité des conditions thermocinétiques liées à l'intermittence de l'insolation efficace, inévitable conséquence de la rotation de la terre.

INFLUENCE DU VENT. - Aux faibles puissances, si l'on emploie deux vitres, la température du collecteur diminue de 3 à $7^{\circ} \mathrm{C}$ avec une accentuation lorsque l'ambiante est plus basse. Aux puissances élevées $\left(900 \mathrm{~W} / \mathrm{m}^{2}\right)$ cette variation passe de 14 à $25^{\circ} \mathrm{C}$ sans que l'ambiante soit modifiée. On imagine l'intérêt d'un pare-vent qui pourrait prendre la forme d'une trémie diffusante dont l'effet serait double : réduction de la turbulence sur la première vitre, en captage de radiations au-delà de la surface vitrée.

L'examen des diagrammes montre que dans les cas extrêmes si l'effet du vent 10 à $0 \mathrm{~m} / \mathrm{s}$ s'ajoute à celui de la variation de l'ambiante $-3^{\circ} \mathrm{C}$ à $+27^{\circ} \mathrm{C}$ pour $G=900 \mathrm{~W} / \mathrm{m}^{2}$ la température d'équilibre du collecteur passe dans les 3 cas cités :

$$
n=
$$

0

$$
1
$$

\section{+9 à $85^{\circ} \mathrm{C} \quad 83$ à $133^{\circ} \mathrm{C} \quad 128$ à $165^{\circ} \mathrm{C} \quad 157$ à $187^{\circ} \mathrm{C}$}

5. Facteur thermostatique d'un insolateur. - Nous avons montré (1) que le bilan thermique, limité au seul rayonnement conduit, pour un appareil donné, à définir une constante thermostatique

$$
\sigma_{1}=\frac{\sigma}{(n+1)(1-r)^{2 n}}=\frac{G}{T_{\mathrm{e}}^{4}}
$$

qui le caractérise à toute température d'équilibre soit pour

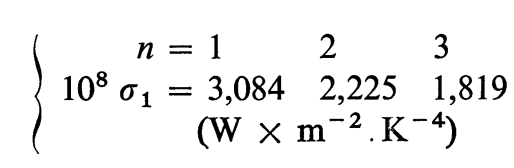

Nous pouvons nous demander si un tel facteur existe lorsque nous tenons compte du rayonnement terrestre lié à l'ambiante et des échanges convectifs externes et internes. Les figures $5 ; 6 ; 7$ donnent $\sigma_{1}$ pour $n=1$; $2 ; 3$ vitres lorsque $G$ varie de 200 à $1000 \mathrm{~W} / \mathrm{m}^{2}, T_{\mathrm{a}}$ vaut $270 \mathrm{~K}$ puis $300 \mathrm{~K}$ et la vitesse du vent passe de 0 à $3 \mathrm{~m} / \mathrm{s}$ puis $10 \mathrm{~m} / \mathrm{s}$.

Résumons les résultats dans le tableau IV où l'on retrouve en l'absence de vent, les valeurs théoriques précédemment calculées. Examinons séparément l'influence des paramètres extérieurs.

Global G. - Deux régimes énergétiques sont à distinguer. A bas régime : $200-500 \mathrm{~W} / \mathrm{m}^{2}$, la convection l'emporte sur le rayonnement et le facteur $\sigma_{1}$ dont la valeur moyenne diminue lorsque $\mathrm{n}$ augmente - ce qui montre l'accentuation de l'effet de serre - ne peut être défini à mieux que 15 à $20 \%$.

Si le global $G$ est élevé soit de 500 à $1000 \mathrm{~W} / \mathrm{m}^{2}$, les effets du rayonnement deviennent prépondérants et $\sigma_{1}$ peut être considéré comme constant avec une précision variant de 3 à $7 \%$.

Température ambiante. - Elle intervient sur le comportement de la vitre externe donc sur les caractéristiques de l'insolateur. Si $T_{\mathrm{a}}$ varie de $270 \mathrm{~K}$ à $300 \mathrm{~K} \sigma_{1}$ diminue d'environ $20 \%$ et la température $T_{\mathrm{e}} \mathrm{du}$ collecteur s'élève d'autant. Tout se passe comme si l'insolateur augmentait son effet de serre. En réalité, ce sont les échanges parasites qui diminuent.

VITESSE DU VENT. - Si elle croît les phénomènes convectifs prédominent au moins pour les faibles valeurs de $G$ et la pente des courbes $\sigma_{1}=f(G)$ augmente ce qui augmente l'incertitude sur $\sigma_{1}$. Si le régime de $\mathrm{G}$ est fort, ces effets s'atténuent d'autant plus que $n$ croît.

Comme un insolateur n'a d'intérêt que pour un rayonnement global assez important il reste possible, dans des conditions climatiques données de définir une constante thermostatique de l'appareil avec une précision de 3 à $7 \%$.

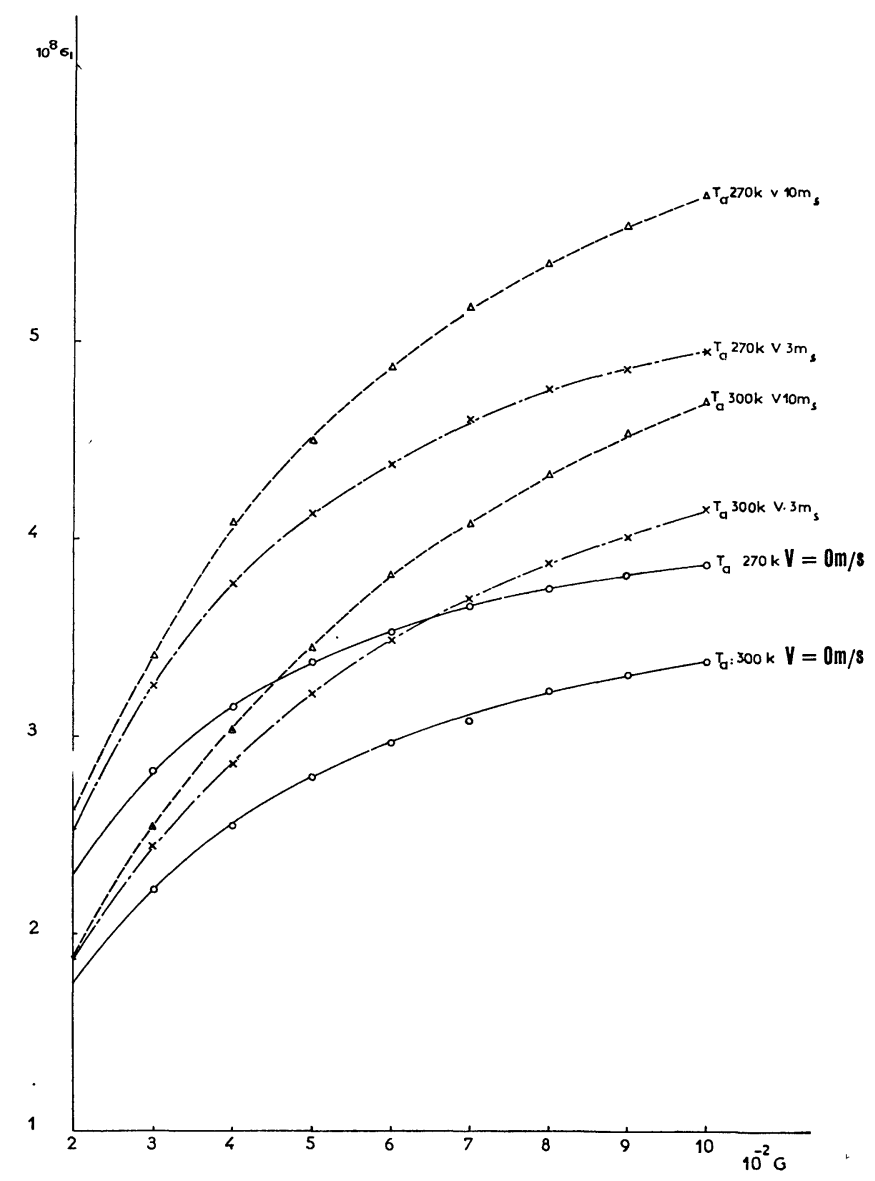

Fig. 5. - Facteur thermostatique d'un insolateur à une vitre en fonction de $G, T_{\mathrm{a}}$ et du vent. 


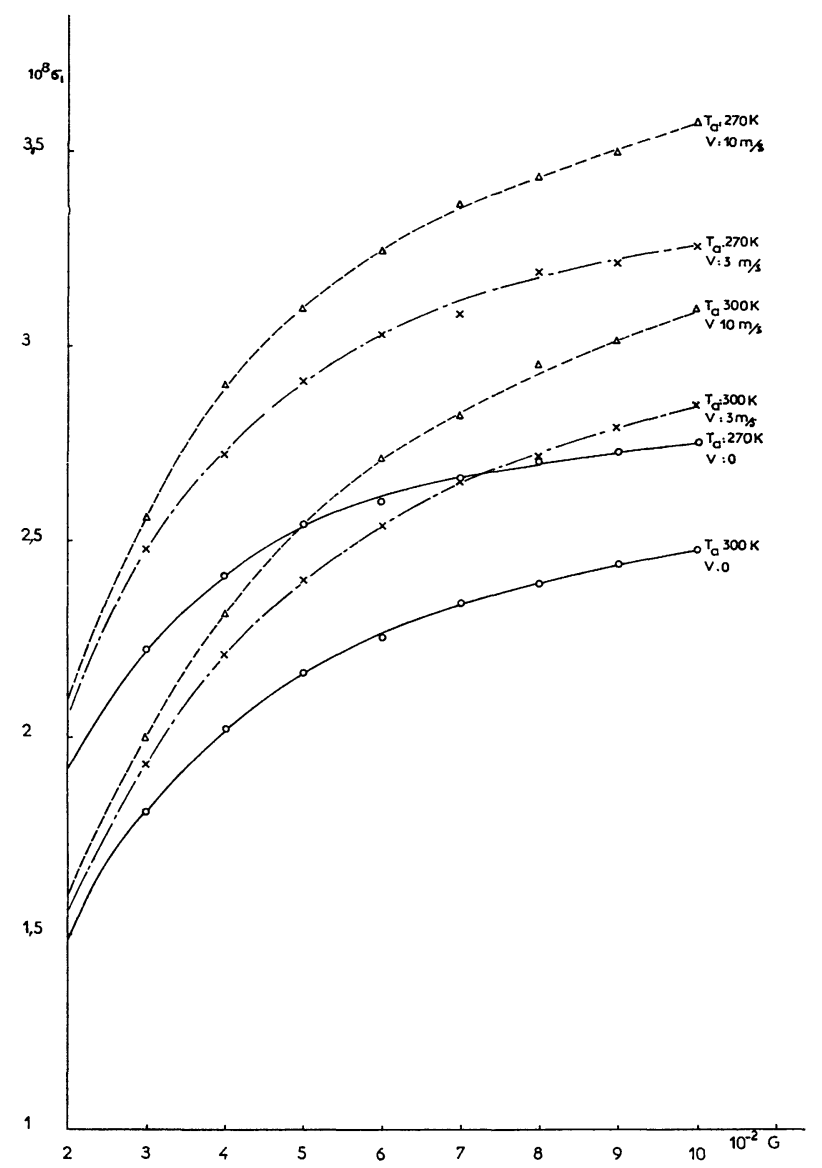

Fig. 6. - Facteur thermostatique d'un insolateur à 2 vitres en fonction de $G, T_{\mathrm{a}}$ et du vent.

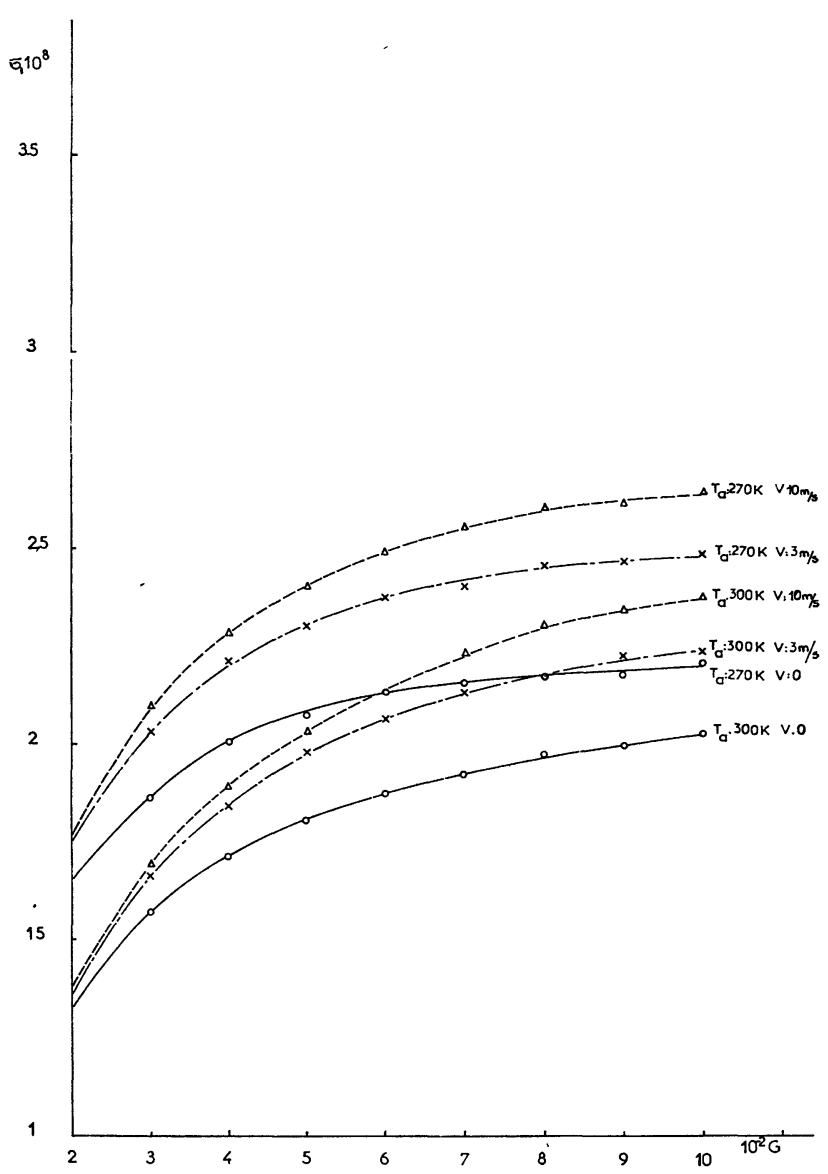

FIG. 7. - Facteur thermostatique d'un insolateur à 3 vitres en fonction de $G, T_{\mathrm{a}}$ et du vent.

\section{TABLEAU IV}

Valeurs moyennes du facteur thermostatique d'un insolateur dans des conditions climatiques diverses $\left(10^{8}\right)$

$$
G: 200-500
$$

$500-1000$

$$
\begin{aligned}
& 1 \text { vitre }: v=0 \\
& \bar{T}_{\mathrm{a}}=270 \mathrm{~K} \\
& v=3 \\
& T_{\mathrm{a}}=300 \mathrm{~K} \\
& T_{\mathrm{a}}=270 \mathrm{~K} \\
& v=10 \\
& T_{\mathrm{a}}=300 \mathrm{~K} \\
& T_{\mathrm{a}}=270 \mathrm{~K} \\
& T_{\mathrm{a}}=300 \mathrm{~K} \\
& 2 \text { vitres }: v=0 \\
& T_{\mathrm{a}}=270 \mathrm{~K} \\
& T_{\mathrm{a}}=300 \mathrm{~K} \\
& v=3 \\
& T_{\mathrm{a}}=270 \mathrm{~K} \\
& v=10 \\
& T_{\mathrm{a}}=300 \mathrm{~K} \\
& T_{\mathrm{a}}=270 \mathrm{~K} \\
& T_{\mathrm{a}}=300 \mathrm{~K} \\
& 3 \text { vitres }: v=0 \\
& T_{\mathrm{a}}=270 \mathrm{~K} \\
& T_{\mathrm{a}}=300 \mathrm{~K} \\
& v=3 \\
& T_{\mathrm{a}}=270 \mathrm{~K} \\
& v=10 \\
& T_{\mathrm{a}}=300 \mathrm{~K} \\
& T_{\mathrm{a}}=270 \mathrm{~K} \\
& T_{\mathrm{a}}=300 \mathrm{~K}
\end{aligned}
$$

$$
\begin{aligned}
& 2,84 \pm 0,53 \\
& 2,27 \pm 0,52 \\
& 3,33 \pm 0,80 \\
& 2,55 \pm 0,68 \\
& 3,57 \pm 0,94 \\
& 2,67 \pm 0,78 \\
& 2,23 \pm 0,31 \\
& 1,83 \pm 0,34 \\
& 2,49 \pm 0,43 \\
& 1,98 \pm 0,42 \\
& 2,60 \pm 0,50 \\
& 2,07 \pm 0,47 \\
& 1,86 \pm 0,21 \\
& 1,57 \pm 0,24 \\
& 2,03 \pm 0,28 \\
& 1,67 \pm 0,31 \\
& 2,11 \pm 0,34 \\
& 1,71 \pm 0,33
\end{aligned}
$$

$3,63 \pm 0,25$

$3,08 \pm 0,29\left({ }^{1}\right)$

$4,54 \pm 0,41$

$3,69 \pm 0,47$

$5,13 \pm 0,63$

$4,07 \pm 0,62$

$2,65 \pm 0,11$

$2,32 \pm 0,16\left({ }^{1}\right)$

$3,08 \pm 0,17$

$2,63 \pm 0,23$

$3,33 \pm 0,24$

$2,80 \pm 0,26$

$2,14 \pm 0,07$

$1,91 \pm 0,11\left(^{1}\right)$

$2,39 \pm 0,09$

$2,11 \pm 0,13$

$2,52 \pm 0,12$

$2,20 \pm 0,17$

(1) Valeurs théoriques calculées précédemment. 
6. Conclusion. - L'intérêt de 2 vitres semble évident du seul point de vue thermostatique. Augmenter le nombre des vitrages (1) conduit même à de meilleures performances: températures d'équilibre plus élevées et plus stables.

Mais nous avons montré (2) que l'intermittence du rayonnement solaire et sa brièveté conduisent à tenir largement compte, pour l'exploitation de cette énergie, des phénomènes de thermocinétique définissant pour un système donné, sa température maximale effectivement atteinte et son rendement. Si nous admettons (2) qu'en première approximation la loi d'échauffemen $t$ d'un insolateur à $n$ vitres, de capacité calorifique constante $M$ est :

$$
\left(T_{\mathrm{e}}-T\right)=\left(T_{\mathrm{e}}-T_{\mathrm{a}}\right)-\frac{k}{M} t
$$

$T_{\mathrm{a}}$ : ambiante $; T_{\mathrm{e}}$ : équilibre. La constante de temps

$$
\tau=\frac{M}{k} \propto M(n+1)
$$

croît avec $n$ puisque,

$$
k=\frac{4 T_{\mathrm{e}}^{3} \sigma}{n+1} .
$$

Il n'est donc pas possible durant une insolation efficace d'approcher suffisamment l'équilibre thermique si le nombre de vitrages dépasse 2 .

Sous flux énergétique fixe, la vitesse de chauffe du collecteur est plus grande si ce dernier comporte une seule vitre et non deux mais, la température limite possible est plus basse ce qui réduit l'intérêt de l'appareil. Tout dépend donc pour le choix d'un type d'insolateur des conditions climatiques, du lieu, et du but recherché.

Signalons enfin, l'importance du facteur thermostatique $\sigma_{1}$ caractérisant un récepteur. Si un vent intense augmente toujours $\sigma_{1}$ en réduisant l'intérêt thermique du capteur plan, notons que dans des conditions habituelles d'utilisation l'ambiante s'élève peu à peu et contribue à stabiliser $\sigma_{1}$. En effet, l'examen des diagrammes montre que si $G$ croît entraînant la croissance de $\sigma_{1}, T_{\mathrm{a}}$ augmente avec comme conséquence une diminution de ce facteur. Sans affirmer qu'il y a compensation entre ces deux variations en sens inverse, il y a bien, du fait de l'expérience, une stabilisation de $\sigma_{1}$. Ceci permettra d'utiliser cette constante en thermocinétique.

\section{Bibliographie}

[1] Flechon, J., Martin, G., Toure, I., C. R. Hebd. Séan. Acad. Sci. B 280 (1975) 211-14.

[2] Flechon, J., Wertwijn, R., Diallo, A., C. R. Hebd. Séan. Acad. Sci. B 231 (1975) 9-12.
[3] Duffie, J. A., Beckman, W. A. Solar Energy Thermal. Hocesses (John Wiley) 1974.

[4] Perrin de Brichambaut, C. Remarques sur l'effet de serre dans les insolateurs plans (1975). 